\title{
REVIEW \\ Ethical practice in Telehealth and Telemedicine
}

\author{
Danielle Chaet, $M S B^{7}$, Ron Clearfield, MD, FACR'2, James E. Sabin, MD'3, and Kathryn Skimming, \\ $M D, M A^{5}$ on behalf of the Council on Ethical and Judicial Affairs American Medical Association
}

${ }^{1}$ American Medical Association, Chicago, IL, USA; ${ }^{2}$, Bonita Springs, FL, USA; ${ }^{3}$ Harvard Pilgrim Health Care, Wellesley, MA, USA; ${ }^{4}$ Harvard Medical School, Boston, MA, USA; ${ }^{5}$ University of Maryland/Sheppard Pratt Psychiatry Residency Program, Baltimore, MD, USA.

This article summarizes the report of the American Medical Association's (AMA) Council on Ethical and Judicial Affairs (CEJA) on ethical practice in telehealth and telemedicine. Through its reports and recommendations, CEJA is responsible for maintaining and updating the AMA Code of Medical Ethics (Code). CEJA reports are developed through an iterative process of deliberation with input from multiple stakeholders; report recommendations, once adopted by the AMA House of Delegates, become ethics policy of the AMA and are issued as Opinions in the Code. To provide enduring guidance for the medical profession as a whole, CEJA strives to articulate expectations for conduct that are as independent of specific technologies or models of practice as possible. The present report, developed at the request of the House of Delegates, provides broad guidance for ethical conduct relating to key issues in telehealth/telemedicine. The report and recommendations were debated at meetings of the House in June and November 2015; recommendations were adopted in June 2016 and published as Opinion E-1.2.12, Ethical Practice in Telemedicine, in November 2016. A summary of the key points of the recommendations can be found in Appendix A (online), and the full text of the opinion can be found in Appendix B (online).

KEYWORDS: ethics; policy; telemedicine; professionalism; AMA (American Medical Association).

J Gen Intern Med 32(10): 1136-40

DOI: $10.1007 / \mathrm{s} 11606-017-4082-2$

(C) Society of General Internal Medicine 2017

I nnovation in information technology is radically changing the ways in which patients perceive time and distance and is reshaping how they interact with and relate to others, including the ways they engage with medicine. As the public becomes increasingly fluent in utilizing novel technologies in all aspects of daily life, evolving applications in health care are altering when, where, and how patients and physicians engage with one another.

Ron Clearfield has retired already

Electronic supplementary material The online version of this article (doi:10.1007/s11606-017-4082-2) contains supplementary material, which is available to authorized users.

Received December 6, 2016

Revised March 23, 2017

Accepted May 3, 2017

Published online June 26, 2017
Prior to recent innovations in information technology, individuals who had a medical concern turned to hardcopy publications, spoke with family or friends, or made an appointment to see their physician. Now, a growing number of these individuals are seeking answers online and can obtain them at virtually any time from virtually anywhere. ${ }^{1}$ Evolving technologies also allow patients to receive care remotely through telemedicine applications, which offer opportunities for patients who are homebound, who live in rural or underserved areas, or who face other impediments that limit their access to care. Likewise, new technologies make it possible for patients who have rare medical disorders to obtain care from distant specialists. ${ }^{2-4}$ Even patients who have access to care in person may find telemedicine a welcome convenience. $^{5}$

While such innovations have significant potential to benefit patients, they also raise ethical challenges. In particular, concerns have been raised that exchanging health information and providing care electronically could create new risks to quality, safety, and continuity of care, all of which could weaken patient-physician relationships. ${ }^{4,6-9}$

\section{TELEHEALTH AND TELEMEDICINE: NEW WAYS TO DELIVER HEALTH CARE}

"Telehealth" and "telemedicine" incorporate technologies and activities that offer new ways to deliver medical care. Although the two are distinguished in current usage, the reasons for doing so are largely administrative. The Health Resources and Services Administration defines "telehealth" broadly as involving electronic and telecommunications technologies to "support and promote longdistance clinical health care, patient and professional health-related education, and public health and administration." 10 For purposes of reimbursement, the Centers for Medicare \& Medicaid Services defines "telemedicine" narrowly as activities involving "two-way, real time interactive communication between the patient and the physician or practitioner at [a] distant site." 11

In telehealth and telemedicine as in other modes of care, patient-physician interactions give rise to differing levels of accountability for physicians. At one end of the telehealth or telemedicine continuum are health-related 
websites where any interaction between an individual seeking health information and a physician who provides it is indirect. The physician has broad obligations to all website users, but is not specifically accountable to any individual information seeker. For example, on some websites, physician experts are responsible for ensuring the accuracy and quality of content, but are not expected to be responsible for how individuals act on the information they find on the website. The analogy is to seeking information from a book or journal article whose author has ensured the accuracy of the content but is not held to account for readers' individual interpretations.

Farther along the continuum are interactions that are more direct, give rise to greater accountability, and carry greater potential for unethical behavior. An example would be an online health website or service where a patient could pose a specific personal health question to which a physician affiliated with the website or service offers an individualized response, which, of course, might include a recommendation to see a physician in person. This interaction might occur in real time or within an established time frame. In such scenarios, the physician, by tailoring the response specifically to the individual, takes on a greater accountability than one who posts general health content for public consumption. This situation might be similar to, though more formal than, a "cocktail party consult" in which a physician is approached for guidance. Disclaimers to the effect that the consultation does not establish a legally recognized patient-physician relationship, which some websites provide, do not obviate the physician's ethical responsibility.

Still farther along the continuum, in a teleradiology or teledermatology consultation, for example, a specialist accesses images that are ideally accompanied by information from the patient's history, reviews them, and offers insight in real time or asynchronously using store-and-forward technology. ${ }^{2}$ The underlying expectation is that the specialist's response will directly inform decisions about the patient's care, for which the specialist will then share accountability with the treating physician in keeping with expectations for in person consultations.

At the far end of the continuum are interactions in which a physician participates directly in a patient's clinical care in real time via telecommunications and is held accountable for the care he or she provides as a treating physician. Telepsychiatry is one example, in which care is electronically mediated, but is not necessarily institutionally based. ${ }^{12}$ Teleoncology provides a second example, in which a specialist provides care for a patient in a remote clinic or other institutional setting, in coordination with on-site professionals involved in the patient's care team. ${ }^{3}$ Physicians are also developing new formats to follow patients with chronic health conditions that take advantage of asynchronous communication to enhance care, provide greater convenience for patients or their surrogates, and enable physicians to make effective use of limited clinical time. ${ }^{13}$

\section{FAMILIAR CHALLENGES, NEW CONTEXT}

Proponents of telehealth and telemedicine highlight how these technologies open new channels of access to care and offer new opportunities for truly patient-centered care. ${ }^{1,5,9,14}$ Critics are more cautious, expressing concern about new or exacerbated risks to privacy and confidentiality, the limitations of electronically mediated interactions for physical examination, and the potential for disruption of the patient-physician relationship. ${ }^{4,7,15,16}$

\section{Risks to Privacy and Confidentiality}

Electronic health and medicine encounters involve a wider range of third parties than traditional health care encounters. Notably, telecommunications service providers and possibly their business affiliates, in addition to health care personnel at one or both ends of the interaction, are involved. Some encounters are protected under privacy laws and regulation, but others may not be protected and may carry additional risks. For example, websites that offer health information may not actually be as anonymous as visitors think; they may leak information to third parties through code on a website or implanted on patients' computers. ${ }^{8}$ Similar concerns may apply to home monitoring devices and mobile health applications to which current privacy protections may not apply. ${ }^{7}$

\section{Matching the Mode of Care to the Patient}

Telemedicine will not be the right model of care for every patient. To begin with, a patient or surrogate must have the resources, including access to and ability to use requisite technology, necessary health care professionals or others present during interactions, access to emergency care, and an acceptable level of comfort in obtaining care in this way. ${ }^{12,17,18}$

Despite its promise, telemedicine is not an appropriate model of care for all medical conditions. ${ }^{4}$ For example, telemedicine is inappropriate for encounters when a hands-on physical examination is crucial or critical data can be gleaned only through direct physical contact. More broadly, telemedicine is not the preferred approach when the technology does not allow physicians to meet established clinical standards.

Whether telemedicine is appropriate for a given patient may also depend on what access the individual otherwise has to health care. For some patients, in some situations, it simply may not be feasible to receive care in person. When the options for a patient are to receive care that may be less than ideal via telemedicine or not to receive care at all, telemedicine services can be appropriate even though the physician, patient, or their surrogate, would prefer that care be provided in person. For example, for a crewmember aboard a submarine or an astronaut in space, telemedicine-whatever its limitations - may be the only way to provide medical services. For a person in an isolated rural setting a 6-h drive from a specialist, telemedicine may be preferable even when an inperson encounter would be marginally superior. 


\section{TRUST AND ETHICAL PRACTICE IN TELEHEALTH AND TELEMEDICINE}

While new technologies and new models of care continue to emerge, physicians' fundamental ethical responsibilities do not change. The practice of medicine is inherently a moral activity, founded in a "covenant of trust" between patient and physician. ${ }^{19}$ In any model of care, patients and their surrogates need to be able to trust that physicians will place patient welfare above other interests (fidelity), provide competent care, provide the information patients and their surrogates need to make well-considered decisions about care (transparency), respect patient privacy and confidentiality, and take steps to ensure continuity of care. ${ }^{20,21}$ The task is to understand how these fundamental responsibilities may play out differently in the context of telehealth and telemedicine than they do in in-person patient-physician interactions.

\section{Fidelity}

The obligation to put patient interests first requires that physicians who participate in telehealth activities or telemedicine programs take steps to minimize conflicts of interest and bias. It is important that physicians disclose financial or other interests that may influence them in their roles with commercial health websites and services and take active steps to manage or eliminate conflicts of interest. ${ }^{22}$

\section{Competence}

The obligation to provide competent care has different implications at different points along the continuum of electronic interactions between physicians and patients or prospective patients. Thus, physicians who provide general health information for online websites have a responsibility to ensure that the content they provide is accurate and objective, just as they would for a professional publication. Physicians who provide personalized responses to individual health queries have additional responsibilities in keeping with their greater accountability to the individual who is seeking guidance. In this context, the obligation of competence requires that the physician who responds to an individual query about a specific health concern have appropriate clinical qualifications and experience and have some means of obtaining the crucial information needed to offer a well-considered professional recommendation. Physicians should bear in mind that state law may further define specific expectations for competence in these situations.

For physicians who provide clinical services, fulfilling the obligation to provide competent care further entails being proficient in the use of the relevant technologies and being comfortable using technology to interact with patients. Competency also includes physicians' responsibility to be aware of the limitations of the telemedicine technologies they use and recognition of limitations in caring for an individual patient. Physicians must use professional judgment in determining what modality of care is best for a given patient, including determining when to shift from telehealth or telemedicine to in-person care.

\section{Transparency and Informed Consent}

Physicians also have a responsibility to be transparent with patients and prospective patients. At one end of the continuum, this may mean no more than disclosing one's credentials as the author of health information. At the other end, it will entail obtaining the patient's informed consent for clinical services that are delivered electronically. In the context of telehealth or telemedicine, patients need to have information not only about medical issues and treatment options, but also about some of the distinctive features of telemedicine.

For example, patients or their surrogates need to have a basic understanding of the credentials of the physicians and other health care professionals who provide telehealth and telemedicine services. Patients also need to be aware of how telemedicine technologies will be used in their care and the limitations of those technologies. Importantly, patients themselves (or their surrogates) or their family members may be asked to play a different role in telemedicine than in traditional care, for example, by learning how to use monitoring devices at home, a factor that may influence decision making. Physicians' responsibility to ascertain whether the patient or family has the skills needed to participate in the care plan may be stronger in the context of telehealth and telemedicine than in other encounters, ${ }^{23}$ especially when telehealth websites or mobile health applications connect physicians and patients with whom there is no prior relationship and or expectation of follow-up.

\section{Privacy and Confidentiality}

The obligation to protect privacy and confidentiality is at least as important in the context of telehealth and telemedicine as in hospital and office settings. Specific responsibilities vary across the continuum of telehealth/telemedicine interactions. Thus, health information websites are expected to publish their privacy policies so that users will know what information is collected from them (if any) and how that information is to be used. ${ }^{24}$ Physicians who provide content for health websites have a responsibility to be satisfied that websites with which they are affiliated have relevant privacy policies. Physicians should refrain from participating in websites that do not make these policies available to website users.

Physicians who answer individual health queries or provide personalized health guidance electronically must be confident that the websites with which they affiliate have appropriate mechanisms in place to protect the confidentiality of individual information exchanged through the website. They should also inform website users that there are potential risks to privacy when personal health information is communicated electronically, for example, through a written disclaimer on the site.

Physicians who provide clinical services via telemedicine must adhere to sound privacy practices themselves and must 
assure themselves that health care professionals at remote websites with whom they collaborate do likewise. They must further assure themselves that the telemedicine services they work with have appropriate protocols to prevent unauthorized access and to protect the security and integrity of patient information. Physicians should alert telemedicine patients or their surrogate that issues of data security and access can arise and inform them of steps taken to protect confidential information.

\section{Continuity of Care}

Fulfilling the obligation not to abandon the patient and to provide for continuity of care ${ }^{20}$ may also take on a new dimension in the context of telemedicine. Physicians who author general health content do not enter into a patientphysician relationship with information seekers; they therefore have no specific responsibilities regarding continuity of care. Physicians who respond to individual health queries should understand that they are responsible for encouraging the patient to seek in-person care when the physician deems that to be needed. Some telehealth and telemedicine services may also identify physicians whom service users can contact to arrange in-person care.

Physicians who provide clinical services through telemedicine should discuss with patients or their surrogates the importance of preserving information for future episodes of care, and whether patients prefer to take responsibility for this or want the physician to do so, by communicating directly with the patient's primary care physician. Information should include recommendations for follow-up care when appropriate. Telemedicine programs that rely on collaboration among the physician, patient, or surrogate and the telemedicine team and that routinely convey the plan to patients' primary physicians if they are not a member of the team are in a better position to develop plans of care that ensure appropriate follow-up. Physicians who provide clinical telehealth and telemedicine services in settings where the encounter will not be documented in an existing medical record should consider writing a note after each clinical encounter for their own files.

\section{THE EVOLVING WORLD OF PATIENT CARE}

Many may feel that telehealth and telemedicine, with their technological sophistication, continuous change, and rapid expansion, are standing medicine on its head. However, it may be more appropriate to see the evolution of telecommunications in patient care as part of the history of technology in medicine, and an opportunity to enhance access to care, quality of care, and satisfaction for both patients and physicians. Thoughtfully implemented, telehealth and telemedicine have the potential to enable physicians to use that most valuable of commodities, time spent in person with patients, to greater effect. $^{13}$
For individuals who are comfortable with electronic technology, telehealth and telemedicine have the potential to increase access to health care by making expert attention available to patients who would otherwise have limited or no access to such care. Yet telehealth and telemedicine cannot enhance access to high-quality care if patients who might benefit from these innovations do not have access to or the ability to use telecommunications technologies effectively. These may include elderly individuals or others who have diminished perceptual, cognitive, or psychomotor abilities ${ }^{23,25}$ or members of communities that tend not to have ready access to or to adopt internet technologies. ${ }^{6,26-29}$ Medicine as a profession can play an important role in advocating for initiatives that will help make the needed technologies more readily available to all patient populations who want to utilize telehealth and telemedicine services.

Achieving the promise and avoiding the pitfalls of electronically mediated care is not the responsibility of individual physicians alone. It requires coordinated effort across the profession, active engagement of specialty and professional organizations not only in medicine but also information technologies, and appropriate education and support for practicing clinicians. ${ }^{15,23}$ The ethical analysis discussed in this article was adopted by the House of Delegates of the American Medical Association, and the recommendations of the report establish ethics guidance in this area. A summary of the key points of the recommendations can be found in Appendix A (online), and the full text of the opinion can be found in Appendix B (online).

Acknowledgements: This article was supported by the American Medical Association. This article summarizes a report written by the Council on Ethical and Judicial Affairs of the American Medical Association and was presented to the AMA's House of Delegates and adopted in June 2016. The authors, on behalf of the Council, are grateful for input and comments provided by members of the AMA during the drafting stages of the report.

Corresponding Author: Danielle Chaet, MSB; American Medical Association, Chicago, IL, USA (e-mail: danielle.chaet@ama-assn.org).

Author Contributions Ms. Chaet, MSB, has access to all of the data used to prepare the report and takes responsibility for the integrity of the interpretation. American Medical Association.

Study Concept and Design.

All authors.

Acquisition of Data.

All authors.

Analysis and Interpretation of Data.

All authors.

Drafting of the Manuscript.

Danielle Chaet.

Critical Revision of the Manuscript for Important Intellectual Content. All authors.

\section{Compliance with Ethical Standards:}

Funding: The study was funded from the operating budget of the American Medical Association, which employs Ms. Chaet.

Conflict of Interest: The authors declare that they have no conflict of interest. 


\section{REFERENCES}

1. Fox S, Duggan M. Health online. 2013. Available at: http://www. pewinternet.org/2013/01/15/health-online-2013/. Accessed 13 April 2017

2. Kvedar J, Coye MJ, Everett W. Connected health: a review of technologies and strategies to improve patient care with telemedicine and telehealth. Health Aff. 2014;33(2):194-199.

3. Doolittle GC, Spaulding AO. Providing access to oncology care for rural patients via telemedicine. J Oncol Pract. 2006;2(5):228-230.

4. Miller TE, Derse AR. Between strangers: the practice of medicine online. Health Aff. 2002;21(4):168-179.

5. Uscher-Pines L, Mehrota A. Analysis of teledoc use seems to indicate expanded access to care for patients without prior connection to a provider. Health Aff. 2014;33(2):258-264.

6. Fleming DA, Edison KE, Pak H. Telehealth ethics. Telemed e-Health 2009; 15(8):797-803.

7. Hall JL, McGraw D. For telehealth to succeed, privacy and security risks must be identified and addressed. Health Aff. 2014;33(2): 216-221.

8. Huesch MD. Privacy threats when seeking online health information. JAMA Intern Med. 2013; 173(19):1838-1840.

9. Agha Z, Schapira RM, Purushottam W, et al. Patient satisfaction with physician-patient communication during telemedicine. Telemed eHealth 2009; 15(9): 830-839.

10. Health Resources and Services Administration. Telehealth. Available at: https://www.hrsa.gov/ruralhealth/telehealth/index.html. Accessed 13 April 2017.

11. Centers for Medicare and Medicaid Services. Telemedicine. Available at: https://www.medicaid.gov/medicaid/benefits/telemed/index.html. Accessed 13 April 2017.

12. American Telemedicine Association. Practice Guidelines for Video-Based Online Mental Health Services. Washington, DC: American Telemedicine Association; 2013.

13. Dixon RF, Rao L. Asynchronous virtual visits for the follow-up of chronic conditions. Telemed e-Health 2014;20(7):1-4.

14. Ackerman MJ, Filart R, Burges LP, et al. Developing next-generation telemedicine tools and technologies: patients, systems, and data perspectives. Telemed e-Health 2009;16(10):93-95.

15. Cotet AM, Benjamin DK. Medical regulation and health outcomes: the effect of the physician examination requirement. Health Econ. 2013;22(4):393-409.
16. Miller EA. The technical and interpersonal aspects of telemedicine: effects on doctor-patient communication. J Telemed Telecare. 2003; 9(1):1-7.

17. American Telemedicine Association. A blueprint for telerehabilitation guidelines. Washington, DC: American Telemedicine Association; 2010.

18. American Telemedicine Association. Home telehealth clinical guidelines. Washington, DC: American Telemedicine Association; 2003.

19. Pellegrino ED. Professionalism, profession and the virtues of the good physician. Mt. Sinai J Med. 2002;69(6):378-384.

20. American Medical Association. Opinion E-10.01, fundamental elements of the patient-physician relationship. Code of Medical Ethics. 2012 Ed. Chicago: American Medical Association; 2012.

21. American Medical Association. Principles of medical ethics. Available at: https://www.ama-assn.org/sites/default/files/media-browser/public/ ethics/principles-of-medical-ethics-20160627.pdf. Accessed 13 April 2017.

22. American Medical Association. Opinion E-5.027, use of health-related online sites. Code of Medical Ethics. 2012 Ed. Chicago: American Medical Association; 2012.

23. Demeris G, Charness $\mathbf{N}$, Krupinski $\mathbf{E}$ et al. The role of human factors in telemedicine. Telemed e-Health 2010;16(4):446-453.

24. Rippen H, Risk A. e-Health Code of Ethics. J Med Internet Res. 2000;2(2):e9. Available at: http://www.ncbi.nlm.nih.gov/pmc/articles/ PMC1761853/. Accessed 13 April 2017.

25. Greysen SR, Garcia CC, Sudore RL, et al. Functional impairment and Internet use among older adults: implications for meaningful use of patient portals. JAMA Intern Med. 2014. Available at: http://archinte. jamanetwork.com/article. aspx?articleid=1873749\&resultclick=3. Accessed 13 April 2017.

26. Fox S. Peer to Peer Health Care; February 28, 2011. Available at: http:// www.pewinternet.org/2011/02/28/peer-to-peer-health-care-2/. Accessed 13 April 2017.

27. Lopez MH. Closing the digital divide: Latinos and technology adoption. 2013. Available at: http://www.pewhispanic.org/2013/03/07/closingthe-digital-divide-latinos-and-technology-adoption/. Accessed 13 April 2017.

28. Smith A. Older adults and technology use. 2014. Available at: http:// www.pewinternet.org/2014/04/03/older-adults-and-technology-use/. 13 Accessed April 2017.

29. Smith A. African-Americans and technology use. 2014. Available at: http://www.pewinternet.org/2014/01/06/african-americans-and-technology-use/. Accessed 13 April 2017. 